\title{
HYDROMAGNETIC FLOW EXTERIOR TO A ROTATING CYLINDER*
}

\author{
By K. B. RANGER (University of Toronto)
}

1. Introduction. Wood [1] has considered the steady incompressible viscous flow between two infinitely long rotating cylinders whose axes are parallel and are slightly displaced so that one cylinder is interior to the other. The flow is developed as a regular perturbation in the small displacement parameter and is such that the leading term is an exact solution of the vorticity equation. It is then possible to solve the first order equations and the interest in the solution is that it represents a viscous flow depending on the Reynolds number. In the problem considered in this paper a similar method is applied to the flow exterior to a rotating cylinder in an infinite conducting fluid where the applied field is produced by an infinite straight wire carrying a steady current and whose axis is parallel to that of the cylinder but slightly displaced from it. The region interior to the cylinder is supposed nonconducting and the wire is fixed and not free to rotate with the boundary of the cylinder. The zero order terms for the flow and magnetic fields are found to be exact solutions of the steady hydromagnetic equations and it is possible, subject to a condition on the flow parameters, to determine the first order terms in a closed form. The interest in the flow is that it represents a hydromagnetic flow depending on the flow parameters $R_{m}$ the magnetic Reynolds number, $R$ the Reynolds number and $M$ the Hartmann number. In fact the functions contained in the first order term in the displacement parameter are simply complex powers of the polar radius whose indices are the roots of a quartic equation. For the two cases in which the magnetic Prandtl number is unity, and for the Stokes flow in which both the Reynolds number and magnetic Reynolds are very small, fairly simple expressions are found for the roots of the quartic equation. It is shown that the solution changes in functional form as the Hartmann number passes through the value two, which also is a feature of the Stokes flow for magnetohydrodynamic Jeffery Hamel solution for motion in a two-dimensional wedge-shaped region. To first order in the displacement parameter it is shown that the magnetic field has no effect on the couple required to maintain the motion. Finally, a generalization to the case of flow between two rotating cylinders is discussed briefly.

2. Flow equations. The steady hydromagnetic flow equations are

$$
\begin{gathered}
(\mathbf{q} \cdot \nabla) \mathrm{q}+\frac{\mu}{\rho}[\mathbf{H} \wedge \operatorname{curl} \mathbf{H}]=-\operatorname{grad} \frac{p}{p}+\nu \nabla^{2} \mathbf{q} \\
\operatorname{curl}[\mathbf{q} \wedge \mathbf{H}]+\eta \nabla^{2} \mathbf{H}=0 \\
\operatorname{div} \mathbf{q}=0, \quad \operatorname{div} \mathbf{H}=0
\end{gathered}
$$

where $\mathbf{q}$ is the fluid velocity, $\mathrm{H}$ the magnetic field, $\rho$ the density, $p$ the pressure, $\mu$ the

${ }^{*}$ Received August 10, 1968; revised version received November 2, 1968. 
permeability, $\nu$ the kinematic viscosity and $\eta$ the diffusivity. For two-dimensional flow flux functions $\psi$ and $\chi$ may be introduced by writing

$$
\mathbf{q}=\operatorname{curl}(-\psi \hat{k}), \quad \mathbf{H}=\operatorname{curl}(-\chi \hat{k})
$$

where $\hat{k}$ is the unit vector directed perpendicular to the plane of motion. On elimination of the pressure, Eqs. (1) and (2) become:

$$
\begin{gathered}
\frac{1}{r} \frac{\partial\left(\psi, \nabla^{2} \psi\right)}{\partial(r, \theta)}-\frac{\mu}{\rho} \frac{\partial\left(\chi, \nabla^{2} \chi\right)}{r \partial(r, \theta)}=\nu \nabla^{4} \psi \\
\frac{1}{r} \frac{\partial(\psi, \chi)}{\partial(r, \theta)}-\eta \nabla^{2} \chi=\mathrm{constant}
\end{gathered}
$$

where $\nabla^{2}$ is the two-dimensional Laplacian given by

$$
\nabla^{2} \equiv \frac{\partial^{2}}{\partial r^{2}}+\frac{1}{r} \frac{\partial}{\partial r}+\frac{1}{r^{2}} \frac{\partial^{2}}{\partial \theta^{2}}
$$

and $(r, \theta)$ are polar coordinates. The flow to be considered is the two-dimensional motion exterior to a circular cylinder of radius $a$ which is rotating with angular velocity $\omega \hat{k}$ in a conducting fluid which is at rest at infinity. The free field originates from an axial current $K \hat{k}$ carried by an infinite straight wire which is parallel to the axis of the cylinder and displaced from it by a small distance $c \ll a$. The position of the wire is fixed and does not rotate with the boundary of the cylinder. When the wire coincides with the axis of the cylinder the flow can be represented by an exact solution of the hydromagnetic equations in which the streamlines and lines of force are systems of concentric circles. The method adopted in the present paper is to perturb about this solution in terms of the small parameter representing the displacement of the axes. If the nondimensional quantities defined by

$$
\psi=\omega a^{2} \psi^{\prime}, \quad \chi=K \chi^{\prime}, \quad \mathbf{r}=a \mathbf{r}^{\prime}, \quad M^{2}=\frac{\mu}{\rho} \frac{K^{2}}{\nu \eta}, \quad R_{m}=\frac{a^{2} \omega}{\eta} \quad R=\frac{a^{2} \omega}{\nu}, \quad \epsilon=c / a
$$

are substituted into the equations of motion, then, on omission of the primes, the scaled equations of flow are

$$
\begin{gathered}
\frac{R}{r} \frac{\partial\left(\Psi, \nabla^{2} \Psi\right)}{\partial(r, \theta)}-\frac{M^{2}}{R_{m} r} \frac{\partial\left(\chi, \nabla^{2} \chi\right)}{\partial(r, \theta)}=\nabla^{4} \Psi \\
\frac{R_{m}}{r} \frac{\partial(\Psi, \chi)}{\partial(r, \theta)}-\nabla^{2} \chi=c
\end{gathered}
$$

where $c$ is a constant. The free field is typified by the flux function $\chi^{0}$, where

$$
\chi^{0}=\frac{1}{2} \log \left(r^{2}-2 r \epsilon \cos \theta+\epsilon^{2}\right)
$$

which to first order in $\epsilon$ may be represented by

$$
\chi^{0}=\log r-\frac{\epsilon}{r} \cos \theta+O\left(\epsilon^{2}\right) .
$$

Thus the wire at $r=\epsilon, \theta=0$ is essentially replaced by a wire at $r=0$, together with a two-dimensional dipole directed along the $x$-axis and of strength $\epsilon$. It is assumed the complete flow and magnetic fields can be expanded in the small parameter $\epsilon$ as 
a power series of the form

$$
\psi=\sum_{n=0}^{\infty} \epsilon^{n} \psi_{n}, \quad \chi=\sum_{n=0}^{\infty} \epsilon^{n} \chi_{n}
$$

If Eq. (10) is substituted into (7) and (8), the zero order equations in $\epsilon$ are the same as (7) and (8) in which $\psi, \chi$ are replaced by $\psi_{0}, \chi_{0}$ respectively. The boundary conditions for the fluid velocity require that

$$
\frac{\partial \psi_{0}}{\partial r}=1, \quad r=1, \quad \frac{\partial \psi_{0}}{\partial r}, \quad \frac{1}{r} \frac{\partial \psi_{0}}{\partial \theta} \rightarrow 0 \quad \text { as } \quad r \rightarrow \infty
$$

and the magnetic field boundary conditions are

$$
\begin{gathered}
\frac{\partial \chi_{0}}{\partial r}=\left(\frac{\partial \chi^{0}}{\partial r}\right)_{\epsilon=0} \quad \frac{1}{r} \frac{\partial \chi_{0}}{\partial \theta}=\left(\frac{1}{r} \frac{\partial \chi^{0}}{\partial \theta}\right)_{\epsilon=0} \text { on } r=1 ; \\
\frac{\partial \chi_{0}}{\partial r}, \quad \frac{1}{r} \frac{\partial \chi_{0}}{\partial \theta} \rightarrow 0 \text { as } r \rightarrow \infty,
\end{gathered}
$$

where it is assumed that the cylinder is nonconducting and possesses the same permeability as the fluid medium. The zero order solutions are readily shown to be

$$
\psi_{0}=\log r, \quad \chi_{0}=\log r
$$

which are in fact exact solutions of the hydromagnetic flow equations (7) and (8) in which the lines of force and streamlines are systems of concentric circles, the magnetic field does not affect the fluid motion, and the magnetic field and velocity field do not interact. Again from (7) and (8) the first order equations in $\epsilon$ are given by

$$
\begin{gathered}
\frac{R}{r} \frac{\partial \psi_{0}}{\partial r} \frac{\partial}{\partial \theta} \nabla^{2} \psi_{1}-\frac{M^{2}}{R_{m} r} \frac{\partial \chi_{0}}{\partial r} \frac{\partial}{\partial \theta} \nabla^{2} \chi_{1}=\nabla^{4} \psi_{1} \\
\frac{R_{m}}{r}\left\{\frac{\partial \psi_{0}}{\partial r} \frac{\partial \chi_{1}}{\partial \theta}-\frac{\partial \chi_{0}}{\partial r} \frac{\partial \psi_{1}}{\partial \theta}\right\}=\nabla^{2} \chi_{1} .
\end{gathered}
$$

The boundary conditions for the fluid velocity require

$$
\frac{\partial \chi_{1}}{\partial r}=\frac{1}{r} \frac{\partial \psi_{1}}{\partial \theta}=0, \quad r=1 ; \quad \frac{\partial \psi_{1}}{\partial r}, \quad \frac{1}{r} \frac{\partial \psi_{1}}{\partial \theta} \rightarrow 0 \quad \text { as } \quad r \rightarrow \infty .
$$

If $\chi_{1}^{i}$ denotes the flux function for the field interior to the cylinder then the field continuity equations are

$$
\frac{\partial \chi_{1}^{i}}{\partial r}=\frac{\partial \chi_{1}}{\partial r}, \quad \frac{\partial \chi_{1}^{i}}{\partial \theta}=\frac{\partial \chi_{1}}{\partial \theta}, \quad r=1
$$

and as $r \rightarrow \infty, \partial \chi_{1} / \partial r,(1 / r) \partial \chi_{1} / \partial \theta \rightarrow 0$. The equation satisfied by $\chi_{1}^{i}$ is

$$
\nabla^{2} \chi_{1}^{i}=0
$$

and for the term of order $\epsilon$ in (9) to be recaptured a suitable form for $\chi_{1}^{i}$ is

$$
\chi_{1}^{i}=\left(a^{1} r-\frac{1}{r}\right) \cos \theta+b^{1} r \sin \theta
$$

where $a^{1}, b^{1}$ are constants. It follows that suitable representations for $\psi$, and $\chi$, are 


$$
\psi_{1}=F_{1}(r) e^{i \theta}, \quad \chi_{1}=G_{1}(r) e^{i \theta}
$$

where it is understood that the real parts are to be taken as the solutions. On substituting (20) into (14) and (15) it is found that $F_{1}$ and $G_{1}$ satisfy the ordinary differential equations

$$
\begin{gathered}
\frac{i R}{r^{2}} D F_{1}-\frac{i M^{2}}{R_{m} r^{2}} D G_{1}=D^{2} F_{1} \\
\frac{i R_{m}}{r^{2}} G_{1}-\frac{i R_{m}}{r^{2}} F_{1}=D G_{1}
\end{gathered}
$$

where $D \equiv d^{2} / d r^{2}+(1 / r)(d / d r)-1 / r^{2}$. In general, there are solutions of (21) and (22) of the form

$$
F_{1}=A_{n} r^{n}, \quad G_{1}=B_{n} r^{n}
$$

where $A_{n}, B_{n}$ and $n$ satisfy the equations

$$
\begin{gathered}
{\left[i R+1-(n-2)^{2}\right]\left(n^{2}-1\right) A_{n}=\frac{i M^{2}}{R_{m}}\left(n^{2}-1\right) B_{n}} \\
i R_{m} A_{n}=B_{n}\left(i R_{m}+1-n^{2}\right) .
\end{gathered}
$$

Eliminating $A_{n}$ and $B_{n}$, it is found that $n= \pm 1$ are solutions provided that $A_{ \pm 1}=B_{ \pm 1}$ and the other values of $n$ satisfy the equation

$$
\left[(n-2)^{2}-(i R+1)\right]\left[n^{2}-\left(i R_{m}+1\right)\right]+M^{2}=0 .
$$

The solutions for $F_{1}$ and $G_{1}$ may then be represented in the form

$$
\begin{aligned}
& F_{1}(r)=A^{1} r+\frac{B^{1}}{r}+\sum_{s=1}^{4} A_{s} r^{n_{s}} \\
& G_{1}(r)=A^{1} r+\frac{B^{1}}{r}+\sum_{s=1}^{4} \frac{i R_{m}}{i R_{m}+1-n_{s}^{2}} A_{s} r^{n}
\end{aligned}
$$

with $A^{1}, B^{1}, A_{s}, s=1,2,3,4$ arbitrary constants and with $n_{s}$ the roots of the quartic equation $f(z)=0$, where

$$
\begin{array}{rl}
f(z)=z^{4}-4 z^{3}+\left(2-i R-i R_{m}\right) z^{2}+4 & z\left(i R_{m}+1\right) \\
& +\left[M^{2}-3-R R_{m}+i\left(R-3 R_{m}\right)\right] .
\end{array}
$$

Now if the outer boundary conditions are to be satisfied it is necessary that at least two roots of $f(z)=0$ have real parts less than unity. Set $z=\zeta+1, \zeta=\alpha+i \beta$; then

$$
g(\zeta)=f(\zeta+1)=\zeta^{4}-\left(4+i R+i R_{m}\right) \zeta^{2}+2 i \zeta\left(R_{m}-R\right)+M^{2}
$$

and employing the argument principle it is found that a necessary and sufficient condition that $g(\zeta)$ has exactly two roots with negative real parts is

$$
\begin{aligned}
h(\beta) & =\operatorname{Re} g(i \beta)=\beta^{4}+4 \beta^{2}-2 \beta\left(R_{m}-R\right)+M^{2} \\
& =\beta^{4}+4\left[\beta-\frac{1}{4}\left(R_{m}-R\right)\right]^{2}+M^{2}-\frac{1}{4}\left(R_{m}-R\right)^{2} \geqq 0
\end{aligned}
$$

for all real $\beta$. A sufficient condition that $h(\beta)$ is positive is clearly $4 M^{2}>\left(R_{m}-R\right)^{2}$. It is noted that if $h(\beta)$ is somewhere negative then it must have at least two real roots 
and consequently $g(\zeta)$ will have at least three roots with negative real parts. In this case the boundary conditions do not determine the term of order $\epsilon$ uniquely as there are eigensolutions and, in principle, the roots have to be combined in such a way so as to ensure the convergence of the complete expansion in $\epsilon$. However, this case will not be considered further in the present work. Thus if $4 M^{2}>\left(R_{m}-R\right)^{2}$, let $n_{i}, i=1,2$, be the roots of (29) satisfying $\operatorname{Re} n_{i}<1$; then the solutions for $F_{1}$ and $G_{1}$ are given by

$$
\begin{aligned}
& F_{1}(r)=B^{1}\left[\frac{2}{r}+\frac{n_{2}+1}{n_{1}-n_{2}} r^{n_{1}}-\frac{n_{1}+1}{n_{1}-n_{2}} r^{n_{2}}\right] \\
& G_{1}(r)=B^{1}\left[\frac{1}{r}+\frac{i R_{m}\left(n_{2}+1\right) r^{n_{1}}}{\left(n_{1}-n_{2}\right)\left(i R_{m}+1-n_{1}^{2}\right)}-\frac{i R_{m}\left(n_{1}+1\right) r^{n_{2}}}{\left(n_{1}-n_{2}\right)\left(i R_{m}+1-n_{2}^{2}\right)}\right] .
\end{aligned}
$$

These solutions are defined for values of the parameters $R, R_{m}$, and $M^{2}$ satisfying the inequality $4 M^{2}>\left(R-R_{m}\right)^{2}$. The remaining constants are determined from the continuity equations (17) and lead to the equations

$b^{\prime} \cos \theta-\left(a^{\prime}-1\right) \sin \theta$

$$
=\operatorname{Re}\left\{B^{1} i e^{i \theta}\left[1+\frac{i R_{m}\left(n_{2}+1\right)}{\left(n_{1}-n_{2}\right)\left(i R_{m}+1-n_{1}^{2}\right)}-\frac{i R_{m}\left(n_{1}+1\right)}{\left(n_{1}-n_{2}\right)\left(i R_{m}+1-n_{2}^{2}\right)}\right]\right\}
$$

$b^{\prime} \sin \theta+\left(a^{\prime}+1\right) \cos \theta$

$$
=\operatorname{Re}\left\{B^{1} e^{i \theta}\left[-1+\frac{i R_{m} n_{1}\left(n_{2}+1\right)}{\left(n_{1}-n_{2}\right)\left(i R_{m}+1-n_{1}^{\overline{2}}\right)}-\frac{i R_{m} n_{2}\left(n_{1}+1\right)}{\left(n_{1}-n_{2}\right)\left(i R_{m}+1-n_{2}^{\overline{2}}\right)}\right]\right\}
$$

so that there are four arbitrary constants $a^{\prime}, b^{\prime}, B^{1}$ and $\bar{B}^{1}$ to satisfy the four linear simultaneous equations arising from (34).

3. Slow motion solution. In this section some explicit values for $n$, will be determined for the case when the motion is slow, that is for effectively zero Reynolds number. If in addition $R_{m}$ is negligibly small and we put $z=\zeta+1$, the equation satisfied by $\zeta$ in the limit as $R_{m}, R \rightarrow 0$ is

$$
\zeta^{4}-4 \zeta^{2}+M^{2}=0 .
$$

Now it is observed that the equation $z^{4}=2 a z^{2}+b^{2}=0$ has the solutions

$$
z= \pm \frac{1}{\sqrt{2}}\left\{(a+b)^{1 / 2} \pm(a-b)^{1 / 2}\right\}
$$

so that the nature of the solutions depends on the sign of $(a-b)$. In the present case $a=2, b=M$. For $0<M<2$, the solutions for $F_{1}(r)$ and $G_{1}(r)$ are given by

$$
\begin{aligned}
& F_{1}(r)=\left\{\frac{1}{r}-\frac{\left(2-\alpha^{-}\right)}{\left(\alpha^{+}-\alpha^{-}\right)} r^{1-\alpha^{+}}+\frac{\left(2-\alpha^{+}\right)}{\left(\alpha^{+}-\alpha^{-}\right)} r^{1-\alpha^{-}}\right\} \\
& G_{1}(r)=-\frac{1}{r}
\end{aligned}
$$

where $\alpha^{ \pm}=\left\{2 \pm\left(4-M^{2}\right)^{1 / 2}\right\}^{1 / 2}$. For $M>2$, the values of $z$ for which $\operatorname{Re} z<1$ are given by 


$$
z=1-\left(\frac{M+2}{4}\right)^{1 / 2} \pm i\left(\frac{M-2}{4}\right)^{1 / 2}
$$

and the solutions are given by

$$
\begin{aligned}
F_{1}(r) & =-\left\{\frac{1}{r}+\frac{\left[2-(M+2 / 4)^{1 / 2}\right]}{(M-2 / 4)^{1 / 2}} r^{1-(M+2 / 4)^{1 / 2}} \sin \left[(M-2 / 4)^{1 / 2} \log r\right]\right. \\
\left.-r^{1-(M+2 / 4)^{1 / 2}} \cos \left[(M-2 / 4)^{1 / 2} \log r\right]\right\} & \\
G_{1}(r) & =-\frac{1}{r} .
\end{aligned}
$$

For $M=2, \zeta=-\sqrt{ } 2$ from (35) and a second solution of the form $r^{1-\sqrt{2}} \log r$ is found from (21). The complete solution for $F_{1}$ satisfying the boundary conditions is given by

$$
F_{1}(r)=-\left\{\frac{1}{r}-r^{1-\sqrt{2}}+(2-\sqrt{ } 2) r^{1-\sqrt{2}} \log r\right\}
$$

and $G_{1}$ is the same as before. Thus (36), (38), and (39) define a magnetohydrodynamic Stokes flow valid for arbitrary $M$. These solutions express the effect which the dipole part of the applied magnetic field has on the fluid motion and this in general is to retard the flow. From (38) the graph of $F_{1}(r)$ against $r$ presents the appearance of a damped wave whose physical effect is to inhibit the flow round the cylinder. The first order term also contains a term which represents a two-dimensional dipole flow located on the axis of the cylinder and is such that for large $M$ it is the dominant part of the field at large distances from the cylinder. It is of interest to note that the solution changes in functional form as $M$ passes through the value 2. Exactly the same situation occurs for the slow motion solution to the magnetohydrodynamic Jeffery Hamel flow [2] in which the motion takes place in a wedge-shaped region and the applied field is produced by an infinite straight wire carrying a steady current placed at the line of intersection of the walls. Finally it is observed that the quartic (30) reduces essentially to a quadratic for the case in which the magnetic Prandtl number $R_{m} / R$ is unity, and consequently the solution for the flow field can be represented in a relatively simple form.

4. The couple on the cylinder. If $q$ and $H$ represent the physical fluid velocity and magnetic field vectors respectively then the couple on the cylinder is given by the formula

$$
\mathbf{G}=\mu \int_{0}^{2 \pi}\{[\mathbf{r} \wedge \mathbf{H}](\mathbf{H} \cdot \hat{r})\}_{r=a} d \theta+\int_{0}^{2 \pi}\left[\mathbf{r} \wedge \mathbf{R}_{r}\right]_{r=a} a d \theta
$$

where the stress vector $\mathbf{R}_{\mathbf{r}}$ is given by

$$
r \mathbf{R}_{\mathbf{r}}=-p \mathbf{r}+\rho \nu[(\mathbf{r} \cdot \nabla) \mathbf{q}-\mathbf{q}+\nabla(\mathbf{q} \cdot \mathbf{r})] .
$$

To first order in $\epsilon$, the magnetic field contribution vanishes and the second integral gives the result

$$
\mathbf{G}=4 \pi a^{2} \rho \nu \omega \hat{k}
$$

so that to first order in $\epsilon$ the magnetic field has no effect on the couple required to maintain the motion. 
5. Flow between two rotating cylinders. It is of interest to enquire whether the preceding analysis applies more generally to the flow between coaxial rotating cylinders whose axes are slightly displaced by a nondimensional distance $\epsilon \ll 1$. Both cylinders are nonconducting and it is assumed that the free field is produced by an infinite straight wire located on the axis of the inner cylinder and carrying a steady current. Proceeding in the same manner as before we reduce the flow problem to the solution of

$$
\begin{aligned}
& i R\left(A+\frac{B}{r^{2}}\right) D F_{1}-\frac{i M^{2}}{R_{m}}\left(C+\frac{D}{r^{2}}\right) D G_{1}=D^{2} F_{1} \\
& i R_{m}\left(A+\frac{B}{r^{2}}\right) G_{1}-i R_{m}\left(C+\frac{D}{r^{2}}\right) F_{1}=D G_{1}
\end{aligned}
$$

where $A, B, C$, and $D$ are constants depending on the radii and angular velocities of the cylinders. The system defined by (42) cannot be solved with the same generality as (21) and (22). A perturbation solution can be obtained easily for small values of all three parameters and for larger values it is possibly more appropriate to employ a boundary layer type of approximation to the system. However, this will not be attempted in the present paper.

\section{References}

[1] W. W. Wood, J. Fluid Mech. 3, 159-177 (1957)

[2] K. B. Ranger, J. Math., and Phys. 46, 1, 21-27 (1967) 